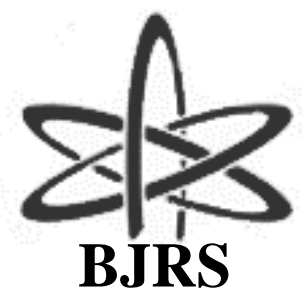

BRAZILIAN JOURNAL

$\mathrm{OF}$

RADIATION SCIENCES

09-01A (2021) 01-09

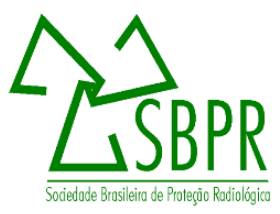

\title{
The influence of the lead eyewear geometry on the doses to the eye lens
}

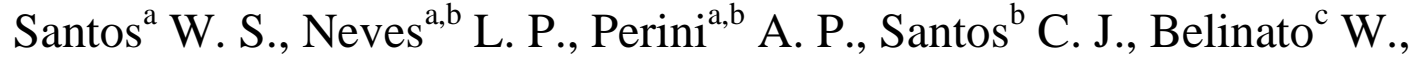 \\ Caldas ${ }^{\mathrm{d}}$ L.V.E. \\ ${ }^{a}$ Instituto de Física - Universidade Federal de Uberlândia (INFIS/UFU), Av. João Naves de Ávila, 2121, 38400-902 \\ Uberlândia, $M G$, Brasil. \\ ${ }^{b}$ Programa de Pós-Graduação em Engenharia Biomédica, Faculdade de Engenharia Elétrica - Universidade Federal \\ de Uberlândia (PPGEB/UFU), Av. João Naves de Ávila 2121, 38400-902 Uberlândia, MG, Brasil. \\ ${ }^{c}$ Departamento de Ensino, Instituto Federal de Educação, Ciência e Tecnologia da Bahia (IFBA), Av. Amazonas, 3150 \\ 45030-220 Vitória da Conquista, BA, Brazil. \\ ${ }^{d}$ Instituto de Pesquisas Energéticas e Nucleares (IPEN / CNEN - SP) Av. Professor Lineu Prestes, 2242, 05508-000 \\ São Paulo, SP, Brasil. \\ william@ufu.br
}

\begin{abstract}
The scattered radiation from interventional procedures is an important source of radiological risk for the medical staff. Considering all affected organs, the eye lens is among the most critical organs. As pointed out by several studies, even for low radiation doses, the appearance of cataract may occur. Considering this scenario, the radiation doses to the eye lens were determined using three different lead eyewear models commonly employed in the interventional radiology. The interventional radiologist was represented by an adult virtual anthropomorphic phantom (MASH3), coupled to the Monte Carlo code MCNP 6.1, in a typical cardiac interventional radiology procedure. The eyewear had a thickness $0.5 \mathrm{mmPb}$ each, and the evaluation was carried out for four different beam angulations (PA, LAO90, LAO65 e RAO65), utilizing a tube voltage of $80 \mathrm{kVp}$, and HVL of $4 \mathrm{mmAl}$. The results pointed out that the shielding efficiency has a strong dependence on the eyewear type utilized, which may be very useful for the decision-making during the acquisition of such equipments.
\end{abstract}

Keywords: Interventional cardiology, Monte Carlo simulation, virtual anthropomorphic phantom. 


\section{INTRODUCTION}

Interventional cardiology (IC) is a modality of interventional radiology (IR), employed by interventional physicians to access the target region in the patient. IR is a medical modality that uses real-time imaging (fluoroscopy) obtained with X-radiation to access the treatment site, usually using a percutaneous access catheter, or employing a contrast agent to visualize radiolucent organs and structures. In the IC procedures, the patients and the medical staff involved are susceptible to receive high radiation doses. This is mainly due to a long time of the procedure, which may sometimes be associated with complications that must be treated during the procedure. Specifically, for the medical team, the radiation doses are strongly correlated to their proximity to the patient, who is the main radiation scattering center, and to the X-ray tube, which emits radiation in different directions. The doses in these procedures vary greatly, as they are affected by several factors, such as irradiation geometry, energy spectra, field size, patient thickness, and others.

Although IC brings enormous benefits to the patient, such as short-term hospitalization and recovery time, reduced risk of contamination compared to surgical procedures, there is also great concern regarding the medical and occupational doses. Even considering a small radiation field, the medical staff is subjected to the scattered radiation, which may lead to lesions in the eye lens [1]. Thus, to make IC procedures safer for the medical staff, a careful dose evaluation of the eye lens should be performed. In this context, the objective of this study was to evaluate occupational exposures in terms of conversion coefficients for the equivalent dose of the eyes and eye lens. Three types of lead eyewear were tested on a virtual anthropomorphic phantom, coupled with the MCNP 6.1 computational code. This approach has been widely used by several studies in the literature since the dose in organs cannot be directly measured [2 - 5].

\section{MATERIALS AND METHODS}

In this study, an adult male virtual anthropomorphic phantom (MASH3) was used [6]. This phantom was modeled based on the ICRP 89 [7]. To perform the simulations, this phantom was coupled to the radiation transport code MCNP 6.1 [8]. In this code, several different particles, 
photons and electrons may be transported individually or coupled (photon/electron) in a threedimensional geometry and heterogeneous system.

To represent a typical configuration of an IC procedure, two MASH3 phantoms were used to represent the interventional radiologist and the patient. The physician was wearing a lead apron, lead eyewear, and a thyroid protector, each of them with an equivalent thickness of $0.5 \mathrm{mmPb}$. $\mathrm{He}$ was positioned $20 \mathrm{~cm}$ from the patient's waist level. The patient was placed in supine on a bed of carbon fiber of dimensions of $70 \times 5 \times 200 \mathrm{~cm}^{3}$. In addition, a set of IC equipment present in a typical room was simulated, such as the surgical instrumentation table, the lead curtain attached to the patient bed, the suspended lead glass, the flat panel detector, and the video monitors. The beam projections and the surface focus distances evaluated were: postero-anterior $(\mathrm{PA} / 60 \mathrm{~cm})$, anterior oblique left and right (LAO65/56 cm and RAO65/53 cm) and left lateral (LAO90/50 cm). This configuration can be seen in Figure 1.

Figure 1. View of the PA (A), RAO65 (B), LAO65 (C), and LAO90 (D) projections of the IC scenario modeled with the MCNP6.1 code, composed of two anthropomorphic phantoms representing the interventional radiologist and the patient on a surgical table. 

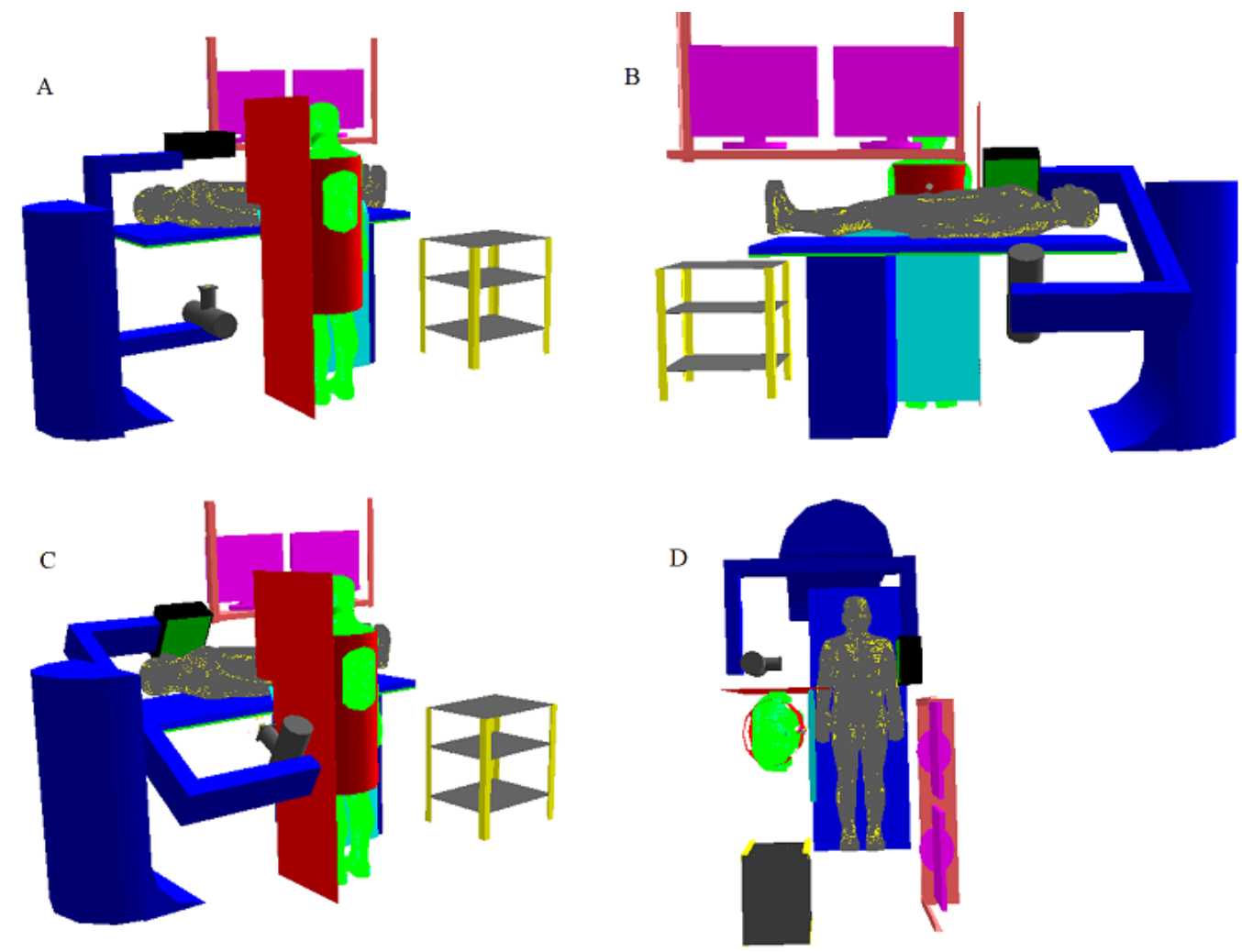

The direct determination of doses in organs and tissues of patients and medical staff, during a clinical procedure, is a complicated and impossible process for most situations. In the literature, involving studies with Monte Carlo simulation and virtual anthropomorphic phantoms, it is common to express the results of doses in organs and tissues by means of the ratio between an estimated or measured dosimetric quantity by another quantity that can be more easily obtained through experimental arrangements such as, for example, dose area product (DAP). The result of this ratio is called the dose conversion coefficient (CC), which was the format used to present the results of this paper.

The materials with chemical and physical characteristics of the virtual antropomorphic phantoms were provided by ICRP 110 [9]. The organs, tissues and other structures of these phantoms are made up of cubic voxels with an edge of $2.24 \mathrm{~mm}$ each.

In this study, a tube voltage of $80 \mathrm{kVp}, 4 \mathrm{mmAl}$ filtration and $12^{\circ}$ anodic angle were used [10]. From this information, it was possible to generate the photon energy spectrum using the SRS 78 software [11]. The X-ray tube was simplified to a point source of photons, directed to irradiate the 
patient's heart, forming a collimated irradiation field in an area of $10 \times 10 \mathrm{~cm}^{2}$. Three types of lead eyewear were molded, with an equivalent lead thickness of $0.5 \mathrm{mmPb}$ each, and with a variable geometric shape. The models were named M1, M2, and M3.

The M1 model has a spherical shape and has an additional piece, a nose bridge, reducing the contact with the physician's face. As a result, it is more comfortable to use. Due to the spherical shape, there is a small space between the spectacle lens and the physician's face. The M2 model represents basic eye protection, and has only one piece with a nose pocket. The M3 model has been shaped to fit closer to the face, and has sides that taper up the sides of the head. This model has a geometric shape of the nose aperture similar but smaller in size than the M1 model and does not have the additional piece around the nose. The three models are shown in Figure 2.

Figure 2. Lead eyewears M1, M2 and M3, positioned in the MASH3 phantom.
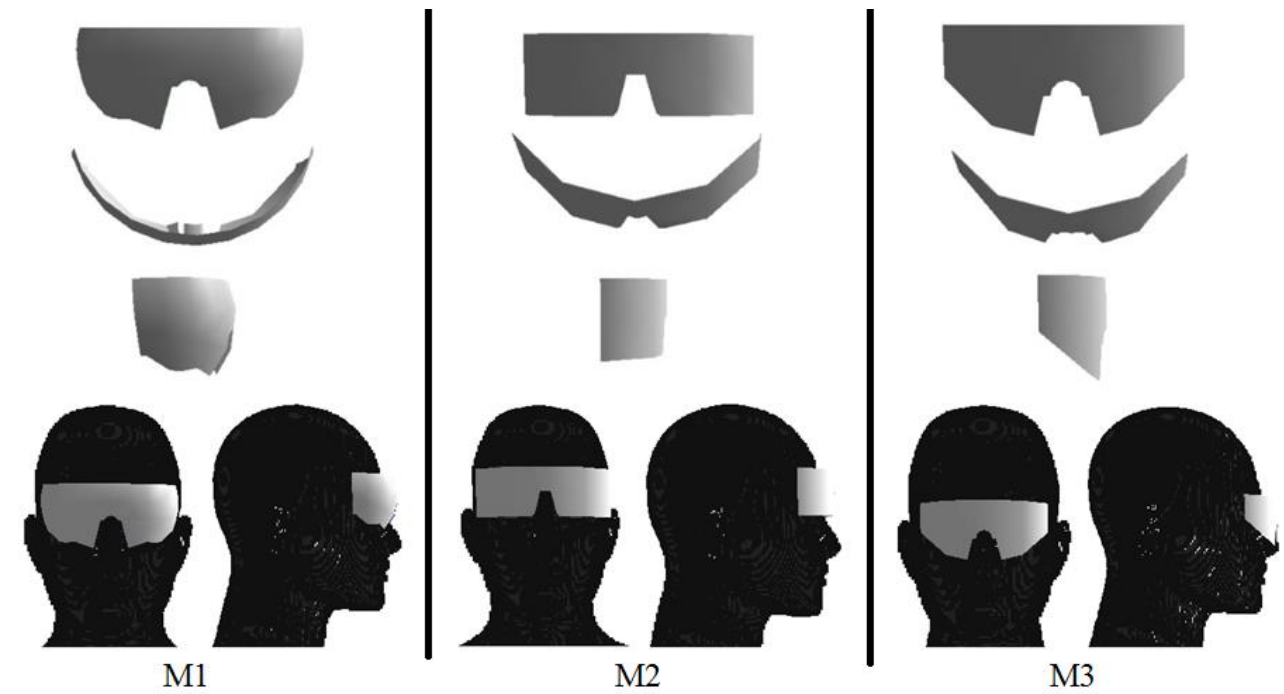

Source: Author

The absorbed doses in each organ and tissue of the MASH3 were calculated using the MCNP 6.1 tally F6 (MeV/g/source-particle). The dosimetric results for the eyes and the eye lens were presented in terms of conversion coefficients (CC), i.e., the ratio of a quantity of interest (equivalent dose, in this case) to a more easily measurable one, as the DAP. The DAP in each projection was calculated from the absorbed dose in an air cell, located $15 \mathrm{~cm}$ at the exit of the X- 
ray tube, multiplied by the area of the beam at this distance. To obtain a satisfactory statistical accuracy, a total of 1E9 photon histories were used in all irradiation scenarios.

\section{RESULTS AND DISCUSSION}

The results, as a function of the projection of the beam and of the lead model, show a significant difference in the protection efficiency of each eyewear model. The differences between the CC values for the equivalent dose of the eye $\left(\mathrm{CC}\left[\mathrm{H}_{\mathrm{T}}\right]_{\text {eye }}\right)$ and eye lens $\left(\mathrm{CC}\left[\mathrm{H}_{\mathrm{T}}\right]_{\text {eye lens }}\right)$ may be observed in Figure 3. For all three models of lead eyewear evaluated, the statistical uncertainty (Type A) associated with the simulation process was less than $3 \%$.

Figure 3. Values of $\mathrm{CC}\left[\mathrm{H}_{T}\right]_{\text {eye }}$ and $\mathrm{CC}\left[\mathrm{H}_{T}\right]_{\text {eye lens }}$ of the interventional radiologist as a function of the projection and of the model of the utilized lead eyewear.
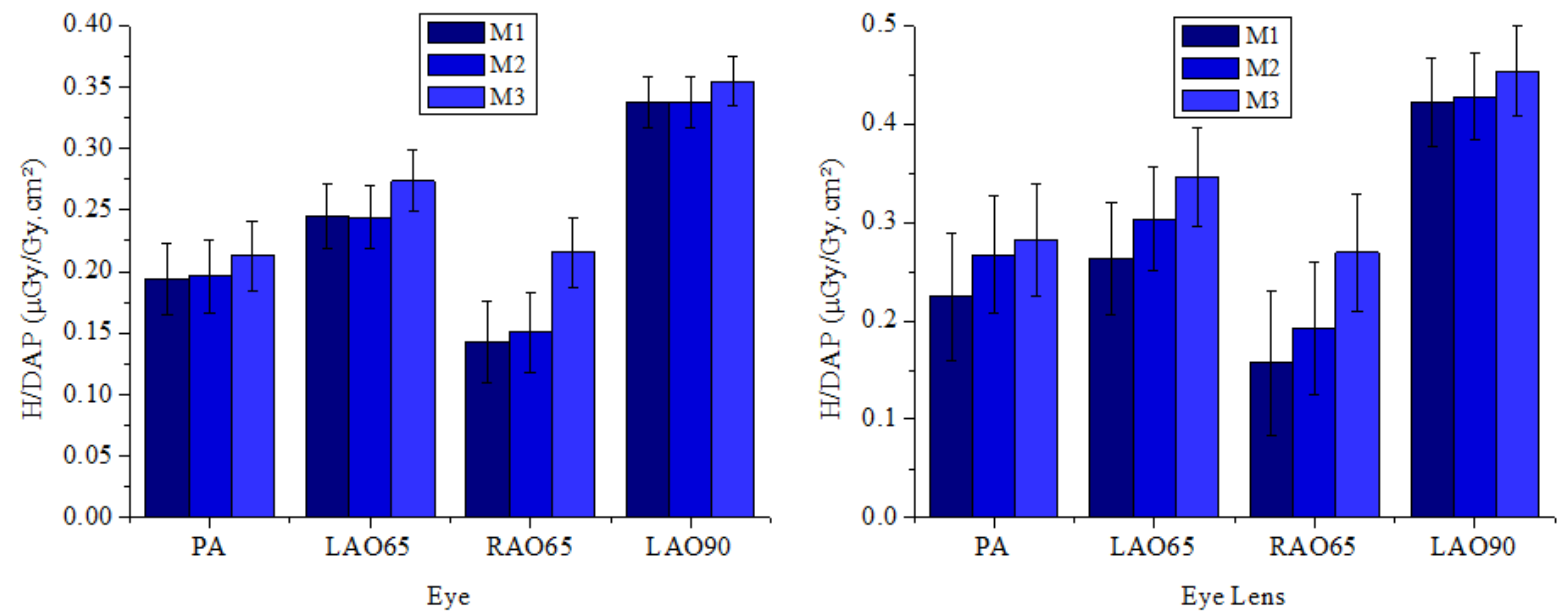

Source: Author

As can be seen in Figure 3, the M1 model protects more efficiently the eyes and eye lens compared to M2 and M3 models. The $\mathrm{CC}\left[\mathrm{H}_{\mathrm{T}}\right]_{\text {eye lens }}$ with the model M1 was $21 \%$ (PA), 24\% (LAO65), 42\% (RAO65), and 7\% (LAO90) lower than those with the M3 model. This can be attributed to the additional shielding material in the opening of the nose since this material probably shielded part of the photons that were scattered by the patient, the patient bed and other equipment 
inside the room that was located in the region of the physician's head. The M3 model presented the lowest shielding efficiency. It has a larger nose aperture than the other two models, and thus allowed more photons to pass through this aperture. The M2 model had less lead material, but still provided better protection, when compared to the M3 model.

In all situations, the most critical projection was the LAO90. In this projection, the highest

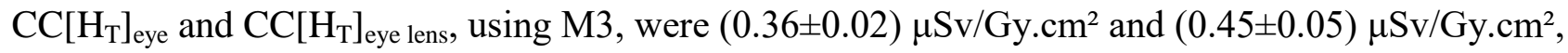
respectively. Considering the most critical situation, that is, when the physician makes use of the M3 model, the $\mathrm{CC}\left[\mathrm{H}_{\mathrm{T}}\right]_{\text {eye lens }}$ using the PA, LAO65, RAO65, and LAO90 projections were 0.28, 0.35, 0.27 and $0.45 \mu \mathrm{Sv} / \mathrm{Gy} . \mathrm{cm}^{2}$, respectively. Considering these projections and an annual workload of 200 coronary angiography procedures, with an average DAP of $33 \mathrm{~Gy} . \mathrm{cm}^{2}$, the dose on the eyes of the interventional physician will be approximately $9 \mathrm{mSv}$, which is in accordance with the effective dose value of $8.8 \mathrm{mSv}$ obtained by [3].

The projection that registered the lowest $\mathrm{CC}$ values was the RAO65. In this projection, the incident beam passes through a greater thickness of the patient, and the X-ray tube is under patient bed, which attenuates the radiation beam. As can be seen in Figure 3, the doses in the eyes and eye lens depend not only on the eyewear model, but also on the directions of the beam. In the LAO90 and LAO65 projections, the X-ray tube is on the right side of the patient and on the same side of the physician, who is exposed to backscattered radiation and, therefore, this projection was responsible for the highest $\mathrm{CC}$ values for the physician.

\section{CONCLUSIONS}

In this study, the $\mathrm{CC}\left[\mathrm{H}_{\mathrm{T}}\right]_{\text {eye }}$ and $\mathrm{CC}\left[\mathrm{H}_{\mathrm{T}}\right]_{\text {eye lens }}$ were presented using virtual anthropomorphic phantoms, named MASH3, to represent an adult patient and an interventional radiologist, coupled to the MCNP6.1 Monte Carlo code. Three types of eyewear geometries, with $0.5 \mathrm{mmPb}$ thickness, were evaluated. The results showed that the geometry of the eyewear interferes with the protection of the eyes and eye lens. In this sense, whenever it is possible, it is important to use devices with a minimal aperture between the face and the eyes, to avoid doses from scattered radiation. Thus, the M1 model showed to be the most effective in relation to the M2 and M3 models, presenting a 
reduction of up to $42 \%$. In addition to the type of lead eyewear used, the direction of the incident beam is an important factor in determining the dose in the eyes and eye lens. The most critical projection for the physician was the LAO90 since it presents greater exposure to the backscattered radiation because in this situation, the physician is closer to the $\mathrm{X}$-ray tube.

\section{ACKNOWLEDGMENTS}

The authors would like to thank Dr. Richard Kramer for kindly providing the virtual anthropomorphic phantoms used in this work. This work was partially supported by the Brazilian agencies: Fundação de Amparo à Pesquisa do Estado de Minas Gerais (FAPEMIG, Grants No. APQ-03049-15 and APQ-02934-15) and Conselho Nacional de Desenvolvimento Científico e Tecnológico (CNPq Grants No. 421603/2016-0, 420699/2016-3 and 301335/2016-8).

\section{REFERENCES}

[1] ICRP 85. INTERNATIONAL COMMISSION ON RADIOLOGICAL PROTECTION. Avoidance of radiation injuries from medical interventional procedures. Oxford, UK, Pergammon Press, Oxford, 2000.

[2] KOUKORAVA, C.; FARAH, J.; STRUELENS, L.; CLAIRAND, I.; DONADILLE, L.; VANHAVERE, F.; DIMITRIOU, P. Efficiency of radiation protection equipment in interventional radiology: a systematic Monte Carlo study of eye lens and whole body doses. J. Radiol. Prot., v. 34, p. 509-528, 2014.

[3] KONG, Y.; STRUELENS, L.; VANHAVERE, F.; VARGAS, C. S,; SCHOONJANS, W.; ZHUO, W. H. Influence of standing positions and beam projections on effective dose and eye lens dose ofanaesthetists in interventional procedures. Radiat. Prot. Dosim., v. 163, p. 181$187,2015$.

[4] FERRARI, P.; BECKER, F.; CARINOU, E.; CHUMAK, V.; FARAH, J.; JOVANOVIC, Z.; KRSTIC, D.; MORGUN, A.; PRINCIPI, S.; TELES, P. Monte Carlo study of the scattered 
radiation field near the eyes of the operator in interventional procedures. J. Radiol. Prot., v. 36, p. 902-921, 2016.

[5] SANTOS, W. S.; BElinATO, W.; PERINI, A. P.; CALDAS, L. V. E.; GALEANO, D. C.; SANTOS, C. J.; NEVES, L. P. Occupational exposures during abdominal fluoroscopically guided interventional procedures for different patient sizes - A Monte Carlo approach. Physica Medica, v. 45, p. 35-43 2018.

[6] CASSOLA, V. F.; DE LIMA, V. J.; KRAMER, R.; KHOURY, H. J. FASH and MASH: Female and male Adult human phantoms based on polygon meSH surfaces. Part II. Dosimetric calculations. Phys Med Biol., v. 55, p. 63-189, 2010.

[7] ICRP 89. INTERNATIONAL COMMISSION ON RADIOLOGICAL PROTECTION. Basic anatomical and physics data for use in radiological protection: reference values. Pergamon Press, Oxford, 2002.

[8] PELOWITZ, D. B. MCNPX User`s Manual. version 2.7.0. Report LA-CP-11-00438, Los Alamos National Laboratory, 2011.

[9] ICRP 110. INTERNATIONAL COMMISSION ON RADIOLOGICAL PROTECTION. Adult Reference Computational Phantoms. Ann ICRP, v.39(2), p. 1-166, 2009.

[10] SANTOS, W. S.; NEVES, L. P.; PERINI, A. P.; BElinATO, W.; CALDAS, L. V. E.; CARVALHO, A. B.; MAIA, A. F. Exposures in interventional radiology using Monte Carlo simulation coupled with virtual anthropomorphic phantoms. Physica Medica, v. 31, p. 929933, 2015.

[11] CRANLEY, K.; GILMORE, B. J.; FOGARTY, G. W. A. L. Catalogue of diagnostic x-ray spectra and other data. Institute of Physics and Engineering in Medicine, New York, USA, 1997. 